\title{
ENERGY-CONSISTENT COROTATIONAL SCHEMES FOR FRICTIONAL CONTACT PROBLEMS*
}

\author{
P. HAURET ${ }^{\dagger}$, J. SALOMON ${ }^{\ddagger}$, A. A. WEISS ${ }^{\S}$, AND B. I. WOHLMUTH ${ }^{\S}$
}

\begin{abstract}
In this paper, we consider the unilateral frictional contact problem of a hyperelastic body in the case of large displacements and small strains. In order to retain the linear elasticity framework, we decompose the deformation into a large global rotation and a small elastic displacement. This corotational approach is combined with a primal-dual active set strategy to tackle the contact problem. The resulting algorithm preserves both energy and angular momentum.
\end{abstract}

Key words. elastodynamic, corotational formulation, energy conservative schemes, large displacements, small strains, contact problem, active set strategy

AMS subject classifications. 35L70, 74B05, 74B20, 74H15

DOI. $10.1137 / 070687827$

1. Introduction. The simulation of contact in elastodynamic problems involving large rigid body motions is a well-known modeling challenge. In particular, the correct definition of a global angle of rotation for an elastic body is not straightforward and requires costly computations. Moreover, the treatment of high rotationalvelocities poses significant issues regarding finite element discretization and the development of suitable time-integration schemes.

Standard geometrically nonlinear formulations handle the situation of large rigid body motion. Nevertheless, once discretized in time, such formulations become unable to render rotations exactly. As a result, when considering high-speed rotation with large time steps, this method exhibits unsatisfying numerical results; the approximation of the rotation is quite bad, and spurious oscillations might be observed. Additionally, even when small strains are considered, the iterative solving procedure remains quite expensive.

In order to solve the aforementioned problem while keeping track of the global rotation of the body, of crucial importance is the decomposition of the movement into large rotations and additional displacements. This idea was first introduced in the pioneering work [2] dealing with aircraft and spacecraft dynamics. The approach was put in an abstract framework by de Veubeke [7], where different criteria were presented to guarantee the uniqueness of the decomposition. Nowadays, the concept is often used by the engineering community, where it is referred to as "corotational formulation." Improvements were given by consistent linearizations $[5,6]$ and element-independent corotational approaches [20,22, 23]. We refer to [9] and [21] for the general overviews of the corotational approach.

${ }^{*}$ Received by the editors April 10, 2007; accepted for publication (in revised form) February 5 , 2008; published electronically July 23, 2008. This work was supported in part by the Deutsche Forschungsgemeinschaft, SFB 404, B8.

http://www.siam.org/journals/sisc/30-5/68782.html

${ }^{\dagger}$ GALCIT, California Institute of Technology, Pasadena, CA 91125 (hauret@cmapx. polytechnique.fr). This author's work was supported by the American Department of Energy through Caltech's ASCI ASAP Center for the Simulation of the Dynamic Response of Materials.

${ }^{\ddagger}$ CEREMADE, Université Paris-Dauphine, Pl. du M ${ }^{a l}$ de Lattre de Tassigny, 75016 Paris, France (julien.salomon@dauphine.fr).

$\S$ Institut für Angewandte Analysis und Numerische Simulation (IANS), Universität Stuttgart, Pfaffenwaldring 57, D-70569 Stuttgart, Germany (weiss@ians.uni-stuttgart.de, wohlmuth@ians.unistuttgart.de). 
Contact adds to the complexity of the situation and has led to many research activities both from the numerical and the theoretical points of view (see $[8,16,19,25$, 27 and the references therein for an overview of the topic). Let us mention here the key difficulty in developing energy-conserving time-integration schemes $[17,1,18,11]$ to handle the nonfrictional components of contact, especially when interested in enforcing simultaneously the persistency and the nonpenetration conditions.

The goal of the present paper is to develop a framework enabling an exact description of rotations, and accurate integration of high-speed rotating elastodynamics with contact. To tackle such problems, we develop a new discretization scheme based on a global corotational formulation and derive an energy and angular momentumpreserving time-integration procedure. Indeed, conservation is crucial regarding longterm accuracy and stability (cf. [12] and references therein).

In the presented approach, we restrict ourselves to the case of large deformations, which are given by a global rotation with fixed rotation axis and global translation, and small strains. A typical application is a rotating wheel. In this setting, the decomposition of the global motion into a rigid part and a purely elastic part allows us to accelerate the computation of the elastic deformation. The efficiency of our method comes from the fact that, in the case of small strains, the local displacements can be handled within the framework of linear elasticity. For the elastodynamic setting, numerical schemes are presented and analyzed in [24]. In this paper, we consider the case including frictional contact. Here, we obtain a nonlinear system where the nonlinearity is restricted to the nodes associated with the contact zone and to the angle of the global rotation. In order to compute contact at each time step, we use a mortar approach with dual Lagrange multipliers [26] and a primal-dual active set strategy as contact solver [15].

The paper is organized as follows. In section 2 , we derive our three-dimensional $(3 D)$ corotational model and present the system of equations describing the evolution of the new unknowns; two variants are proposed, based upon various linearizations. Section 3 provides energy- and momenta-conserving time-discretization schemes for the preceding formulations. Space discretization is introduced in section 4, and section 5 presents the algorithms exploited to solve the fully discretized systems. Finally, in section 6 , we show the efficiency and flexibility of our approach through various numerical tests.

2. Model. In this section, we introduce, analyze, and discuss two corotational formulations for the elastodynamic evolution of a single elastic body submitted to frictional contact. After recalling the standard dynamic setting in the presence of hyperelastic materials, we introduce a corotational decomposition of the deformation and derive the equations to which the new unknowns obey. Assuming the body undergoes large rotations but small strains, the decomposition allows us to use a linear elastic material law; only the rotation angle is described by a nonlinear equation. Energy and angular momentum conservation are shown. As the angular equation is linearized, a second model is derived; unlike energy, angular momentum is no more preserved.

In what follows, the following notation is used:

$$
\dot{a}(x, t):=\frac{\partial}{\partial t} a(x, t), \quad \ddot{a}(x, t):=\frac{\partial^{2}}{\partial t^{2}} a(x, t) .
$$

$A^{\top}$ stands for the transpose of matrix $A$, and $\operatorname{tr}(A)$ for its trace. The identity matrix in $\mathbb{R}^{3}$ is denoted by $I, R_{\theta}^{\Delta}$ is the matrix in $\mathbb{R}^{3 \times 3}$ representing the rotation of axis 
$\Delta \in \mathbb{R}^{3}$ and angle $\theta$ as oriented by $\Delta$. The tangent rotation matrix at $\theta=0$ is $\Pi^{\Delta}=\left.\partial_{\theta} R_{\theta}^{\Delta}\right|_{\theta=0}$; for every $x \in \mathbb{R}^{3}$, one has $\Pi^{\Delta} x=\frac{\Delta}{|\Delta|} \times x$. The usual matricial scalar product between the matrices $A, B \in \mathbb{R}^{3 \times 3}$ is $A: B=\operatorname{tr}\left(A^{\top} B\right)$, and "." denotes the usual scalar product in $\mathbb{R}^{3}$.

2.1. One-body frictional contact problem. Let $\Omega \subset \mathbb{R}^{3}$ be a bounded open domain with a piecewise smooth boundary $\Gamma:=\partial \Omega$ representing the reference configuration of a hyperelastic body of mass density $\rho$. The coordinate system is chosen such that the center of gravity has zero coordinates, i.e., $\int_{\Omega} \rho x=0$. Moreover, we consider a fixed obstacle occupying the domain $\Omega_{r}$ with piecewise smooth boundary $\Gamma_{r}:=\partial \Omega_{r}$.

The boundary of the domain $\Omega$ is divided into two disjoint open sets as $\Gamma=\Gamma_{N} \cup$ $\Gamma_{C}$. Neumann conditions are prescribed on $\Gamma_{N}$. The set $\Gamma_{C}$ is the boundary region of the body where contact with the obstacle can occur. Let $\varphi: \Omega \times[0, T] \rightarrow \mathbb{R}^{3}$ be the deformation mapping, and let $d:=\varphi-x$ be the corresponding field of displacements. The deformation gradient is denoted by $F=\nabla \varphi$, and the Green-Saint Venant strain tensor $E(d)$ is given by

$$
E(d):=\frac{1}{2}\left((\nabla \varphi)^{\top}(\nabla \varphi)-I\right)=\frac{1}{2}\left(\nabla d+\nabla d^{\top}+\nabla d^{\top} \nabla d\right) .
$$

Under the assumption of frame indifference, the stored energy density $W$ of the hyperelastic material at a given location $x \in \Omega$ is necessarily a function of this sole strain tensor (cf. Ciarlet [4]). The second Piola-Kirchhoff stress tensor $\Sigma$ is defined by

$$
\Sigma:=\frac{\partial W}{\partial E}(E(d))
$$

and the Cauchy stress $\sigma$ by

$$
\sigma:=\frac{1}{\operatorname{det} F} F \Sigma F^{\top} .
$$

To express contact conditions, we define the normal pressure $\sigma_{\nu}$ and the tangential force $\sigma_{\tau}$ by

$$
\sigma_{\nu}:=(\sigma \nu) \cdot \nu, \quad \sigma_{\tau}:=\sigma \nu-\sigma_{\nu} \nu
$$

respectively, and the normal and tangential displacements by

$$
d_{\nu}:=d \cdot \nu, \quad d_{\tau}:=d-d_{\nu} \nu
$$

Above, $\nu$ stands for the outer normal vector of the deformed domain $\varphi(\Omega)$.

In order to formulate the nonpenetration condition between the two bodies, we introduce a gap function $\gamma_{C}: \Gamma_{C} \rightarrow \mathbb{R}$ that models the distance between the deformed location of a point belonging to $\Gamma_{C}$ and its projection onto $\Gamma_{r}$ along the normal $\nu$, i.e.,

$$
\gamma_{C}:=\left(\pi_{\nu} \varphi-\varphi\right) \cdot \nu,
$$

where $\pi_{\nu}$ is the projection along the normal vector, as illustrated in Figure 2.1.

The nonpenetration of $\Gamma_{C}$ into $\Omega_{r}$ expressed by the Karush-Kuhn-Tucker conditions is formulated as:

$$
\gamma_{C} \geq 0, \quad \sigma_{\nu} \leq 0, \quad \sigma_{\nu} \gamma_{C}=0
$$




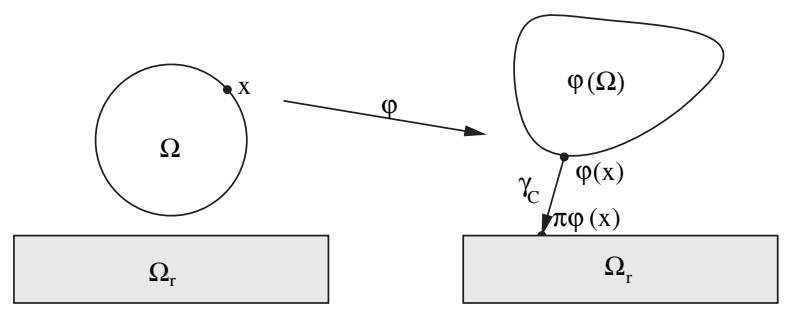

FIG. 2.1. A definition of the gap function $\gamma_{C}$.

Here, the first condition corresponds to the nonpenetration constraint. The last condition implies that the obstacle only applies an effort on the boundary of the domain where contact is active, i.e., $\gamma_{C}=0$. Observe that, for small displacements $d$, the gap function $\gamma_{C}$ can be approximated by $\gamma_{C} \approx \gamma_{C, 0}-d_{\nu}$ where $\gamma_{C, 0}(x)=\pi_{\nu_{0}} x-x$ is the initial distance; $\nu_{0}$ denotes the outer normal vector of $\Omega$. Then, the condition $\gamma_{C} \geq 0$ reads $d_{\nu} \leq \gamma_{C, 0}$, the function $\gamma_{C, 0}$ being constant in time.

In case of contact, we obtain $\pi_{\nu} \varphi=\varphi$. As this condition is fulfilled, contact is said to be persistent if normal velocity vanishes as long as the contact pressure remains positive, which reads

$$
\sigma_{\nu} \dot{d}_{\nu}=0
$$

The seminal contribution from Laursen and Chawla [17] has established that the persistency condition (2.4) is key as far as energy conservation is concerned. Nevertheless, for most numerical schemes, conditions (2.3) and (2.4) cannot be fulfilled simultaneously. For energy conservation purposes, the authors enforce a discretized version of (2.4), at the price of violating (2.3) at second order with respect to the time step.

Frictionless contact is characterized by a null tangent force $\sigma_{\tau}=0$ on $\Gamma_{C}$. In the presence of Coulomb's friction with friction coefficient $\mathfrak{F}$, the tangent force is given by

$$
\left|\sigma_{\tau}\right|-\mathfrak{F}\left|\sigma_{\nu}\right| \leq 0, \quad \dot{d}_{\tau}+\beta^{2} \sigma_{\tau}=0, \quad \dot{d}_{\tau}\left(\left|\sigma_{\tau}\right|-\mathfrak{F}\left|\sigma_{\nu}\right|\right)=0 .
$$

The first equation above provides a bound of the tangential stress as a function of the contact pressure, the second equation enforces a tangential stress opposite to the tangential velocity, and the last equation implies that the contact is either sticky, i.e., tangential velocity vanishes, or slippy if the tangential stress reaches the maximum given in terms of the normal stress.

Let us now formulate the evolution equations of the contact problem under investigation. The body is subjected to volume forces $f(x, t)$ and to boundary tractions $g(x, t)$ on $\Gamma_{N}$. We denote by $\tilde{\lambda}$ the Lagrange multiplier representing the contact forces $-\sigma \nu . \mathcal{V}$ stands for the space of displacements, and the space $\mathcal{M}$ of Lagrange multipliers is the dual space of the trace space $\mathcal{W}$ of $\mathcal{V}$ restricted to $\Gamma_{C}$. The mixed formulation of the problem reads as follows.

Find $(d, \tilde{\lambda})$ such that, for almost every time $t \in[0, T], d(t) \in \mathcal{V}, \tilde{\lambda}(t) \in \mathcal{M}(\tilde{\lambda})$ and

$$
\begin{aligned}
m(\ddot{d}, \eta)+a_{n l}(d, \eta)+b(\eta, \tilde{\lambda}) & =F(\eta), & & \eta \in \mathcal{V}, \\
b_{\tau}(\dot{d}, \mu-\tilde{\lambda}) & \leq\left\langle\gamma_{C}, \mu_{\nu}-\tilde{\lambda}_{\nu}\right\rangle, & & \mu \in \mathcal{M}(\tilde{\lambda}),
\end{aligned}
$$

with the convex set of admissible multipliers

$$
\mathcal{M}(\lambda):=\left\{\mu \in \mathcal{M}:\langle\mu, \xi\rangle \leq \mathfrak{F}\left\langle\lambda_{\nu},\left|\xi_{\tau}\right|\right\rangle \text { for any } \xi \in \mathcal{W} \text { such that } \xi_{\nu} \leq 0\right\} .
$$


We have used the following notation

$$
\begin{gathered}
m(\ddot{d}, \eta):=\int_{\Omega} \rho \ddot{d} \cdot \eta, \quad a_{n l}(d, \eta):=\int_{\Omega} \frac{\partial W}{\partial E}(E(d)):\left((I+\nabla d)^{\top} \nabla \eta\right), \\
b(\eta, \mu):=b_{\nu}(\eta, \mu)+b_{\tau}(\eta, \mu):=\left\langle\eta_{\nu}, \mu_{\nu}\right\rangle+\left\langle\eta_{\tau}, \mu_{\tau}\right\rangle:=\int_{\Gamma_{C}} \eta_{\nu} \cdot \mu_{\nu} d s+\int_{\Gamma_{C}} \eta_{\tau} \cdot \mu_{\tau} d s,
\end{gathered}
$$

and

$$
F(\eta):=\int_{\Omega} f \cdot v+\int_{\Gamma_{N}} g \cdot v d s
$$

Observe that, for small displacements, the contact condition can be approximated by

$$
b_{\nu}(d, \mu-\tilde{\lambda})+b_{\tau}(\dot{d}, \mu-\tilde{\lambda}) \leq\left\langle\gamma_{C, 0}, \mu_{\nu}-\tilde{\lambda}_{\nu}\right\rangle, \quad \mu \in \mathcal{M}(\tilde{\lambda}) .
$$

Doing so, the new right-hand side in (2.6) becomes independent of the deformation.

2.2. Corotational formulation and linear elasticity. Linearized elasticity assumes that both displacements and strains are small. Small strains imply that the approximation

$$
\frac{\partial W}{\partial E}(E(d)) \approx \frac{\partial^{2} W}{\partial E^{2}}(0): E(d)
$$

in the case of isotropic materials, the latter expression reduces to the Saint VenantKirchhoff law (cf. Ciarlet [4]). Furthermore, the assumption of small displacements entails that

$$
E(d) \approx \varepsilon(d):=\frac{1}{2}\left((\nabla d)^{\top}+\nabla d\right) .
$$

Of course, when large rotations are considered, the assumption of small displacements is violated even though strains remain small, and linearized elasticity loses any interest.

For simplicity, we assume here that the body under consideration will undergo large rotations with respect to a single axis, of direction $\Delta$, fixed once and for all. The corotational formulation decomposes the deformation as follows:

$$
\varphi(x, t)=R_{\theta}(t)(x+u(x, t)),
$$

where $R_{\theta}(t)$ is a global rotation of axis $\Delta$ and angle $\theta$, and $u(x, t)$ the field of displacements with respect to the rotated configuration. In order to guarantee the uniqueness of the decomposition (2.8), an additional constraint has to be considered. Following the approach of de Veubeke [7], $\varphi$ being given, we choose $\theta$ so that the weighted $L^{2}$-norm of $u$, namely $\int_{\Omega} \rho u^{2}$, be minimal. This minimization condition is equivalent $[10]$ to

$$
\int_{\Omega} \rho u \cdot \Pi x=m(u, \Pi x)=0 .
$$

Here, $\Pi$ denotes the tangent rotation matrix $\Pi^{\Delta}$ for the fixed rotation axis $\Delta$. Consequently $u$ is free from any elementary rotation. We are thus looking for the displacement $u$ in the space:

$$
\mathcal{X}:=\left\{u \in \mathcal{V}, \int_{\Omega} \rho u \cdot \Pi x=0\right\}
$$

Copyright $@$ by SIAM. Unauthorized reproduction of this article is prohibited. 
We remark that another possible decomposition is splitting the deformation into translation, rotation, and displacement. Indeed, in our decomposition, we do not isolate explicitly large translations, unlike [7], as the new displacement $u$ can include global translations.

However, the underlying translation can be computed in a postprocess by

$$
T(u):=\frac{\int_{\Omega} \rho u}{\int_{\Omega} \rho} .
$$

Observe that the operator $T$ commutes with the operator $\Pi$, i.e., $T(\Pi u)=\Pi T(u)$ and satisfies the following property:

$$
m(T(a), b)=m(T(a), T(b)), \quad a, b \in \mathcal{V} .
$$

Then, the decomposition into a global rigid body motion and a local deformation is given by

$$
\varphi=\tilde{T}+R_{\theta}(x+\tilde{u}),
$$

with $\tilde{u}:=u-T(u)$ and $\tilde{T}:=R_{\theta} T(u)$ and $(\theta, u)$ are given by decomposition (2.8). Hence, we can use our decomposition into rotation and displacements (including translations) and postprocess the full decomposition into rigid body motion and translation-free displacements.

The decomposition (2.8) has the important following property:

$$
E(d)=E(u),
$$

where $E$ is the large strain as defined in (2.1). Since the large rotation part has been filtered out of $d$, the rotated field of displacements $u$ has small amplitude up to the addition of a uniform translation. It becomes possible to make the following approximation:

$$
E(d) \approx \varepsilon(u)
$$

Let us now rewrite the weak mixed formulation (2.6) in terms of the new variables $(\theta, u)$. Using $(2.13)$ and $x+d=R_{\theta}(x+u)$ in the definition of $a_{n l}$, the elastic term becomes

$$
a_{n l}(d, \eta)=\int_{\Omega} \frac{\partial W}{\partial E}(E(u)):\left((I+\nabla u)^{\top} R_{\theta}^{\top} \nabla \eta\right)=a_{n l}\left(u, R_{\theta}^{\top} \eta\right) .
$$

In order to rewrite the acceleration term, we define the relative velocity by

$$
s(x, t):=R_{\theta(t)}^{\top} \dot{\varphi}(x, t)=\dot{u}(x, t)+\dot{\theta}(t) \Pi r(x, t),
$$

with

$$
r(x, t):=x+u(x, t) .
$$

The acceleration term can then be expressed by

$$
\ddot{d}(x, t)=\ddot{\varphi}(x, t)=R_{\theta(t)}(\dot{s}(x, t)+\dot{\theta}(t) \Pi s(x, t)),
$$

Copyright $@$ by SIAM. Unauthorized reproduction of this article is prohibited. 


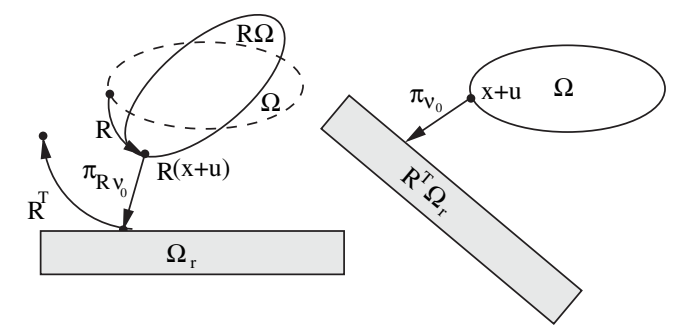

FIG. 2.2. Rotated projection. Left: Operator $R_{\theta}^{\top} \pi_{R_{\theta} \nu_{0}} R_{\theta}$. Right: Operator $\pi_{\nu_{0}}^{\theta}$.

hence

$$
m(\ddot{d}, \eta)=m\left(\dot{s}+\dot{\theta} \Pi s, R_{\theta}^{\top} \eta\right) .
$$

We finally rewrite the frictional contact formulation. As we consider a motion close to a rigid body motion, we assume that the normal vector can be approximated by

$$
\nu \approx R_{\theta} \nu_{0}
$$

We first consider the nonpenetration condition $\gamma_{C} \geq 0$. Using the approximation (2.18) in the definition $(2.2)$ of $\gamma_{C}$, we get

$$
\begin{aligned}
\gamma_{C}(x) & \approx\left(\pi_{R_{\theta} \nu_{0}} R_{\theta}(x+u)-R_{\theta}(x+u)\right) \cdot R_{\theta} \nu_{0} \\
& =\left(R_{\theta}^{\top} \pi_{R_{\theta} \nu_{0}} R_{\theta}(x+u)-(x+u)\right) \cdot \nu_{0} \\
& =\left(R_{\theta}^{\top} \pi_{R_{\theta} \nu_{0}} R_{\theta}(x+u)-x\right) \cdot \nu_{0}-u_{\nu_{0}},
\end{aligned}
$$

where $u_{\nu_{0}}:=u \cdot \nu_{0}$. To simplify this expression, we first take a closer look to the operator $R_{\theta}^{\top} \pi_{R_{\theta} \nu_{0}} R_{\theta}$. Here, a rotated point is projected in the direction of a rotated vector onto the obstacle. The resulting point is then back-rotated. Applying a rotation, we see that this is equivalent (see Figure 2.2) to a projection with respect to $\nu_{0}$ onto the back-rotated obstacle, i.e.,

$$
R_{\theta}^{\top} \pi_{R_{\theta} \nu_{0}} R_{\theta}=\pi_{\nu_{0}}^{\theta}
$$

where $\pi_{\nu_{0}}^{\theta}$ is the projection onto $R_{\theta}^{\top}\left(\Omega_{r}\right)$ in direction $\nu_{0}$. We finally get

$$
\gamma_{C}(x)=\gamma_{C, \theta}-u_{\nu_{0}}
$$

where the function $\gamma_{C, \theta}$ is defined by $\gamma_{C, \theta}:=\left(\pi_{\nu_{0}}^{\theta}(x+u)-x\right) \cdot \nu_{0}$. We remark that, in the case $x+u \approx x$, we obtain

$$
\gamma_{C, \theta} \approx\left(\pi_{\nu_{0}}^{\theta} x-x\right) \cdot \nu_{0}
$$

leading to a right-hand side which only depends on $\theta$. Using this computation of the gap function, the nonpenetration conditions $(2.3)$ read

$$
u_{\nu_{0}} \leq \gamma_{C, \theta}, \quad \tilde{\sigma}_{\nu_{0}}(u) \leq 0, \quad \tilde{\sigma}_{\nu_{0}}(u)\left(u_{\nu_{0}}-\gamma_{C, \theta}\right)=0,
$$

where $\tilde{\sigma}=R_{\theta}^{\top} \sigma R_{\theta}$. Defining $s_{\tau_{0}}:=s-\left(s \cdot \nu_{0}\right) \nu_{0}$ and $\tilde{\sigma}_{\nu_{0}}:=\left(\tilde{\sigma} \nu_{0}\right) \cdot \nu_{0}, \tilde{\sigma}_{\tau_{0}}:=$ $\tilde{\sigma} \nu_{0}-\tilde{\sigma}_{\nu_{0}} \nu_{0}$, the friction conditions (2.5) can be rewritten as

$$
\left|\tilde{\sigma}_{\tau_{0}}(u)\right|-\mathfrak{F}\left|\tilde{\sigma}_{\nu_{0}}(u)\right| \leq 0, \quad s_{\tau_{0}}+\beta^{2} \tilde{\sigma}_{\tau_{0}}(u)=0, \quad s_{\tau_{0}}\left(\left|\tilde{\sigma}_{\tau_{0}}(u)\right|-\mathfrak{F}\left|\tilde{\sigma}_{\nu_{0}}(u)\right|\right)=0 .
$$

Copyright ( by SIAM. Unauthorized reproduction of this article is prohibited. 
Concerning the persistency condition (2.4), we obtain

$$
\tilde{\sigma}_{\nu_{0}}(u) s_{\nu_{0}}=0
$$

with $s_{\nu_{0}}:=s \cdot \nu_{0}$. These conditions are the standard contact and friction conditions with a fixed normal vector. However, for our setting, we have to use a modified gap function, and have in mind that the conditions are formulated in terms of $R_{\theta}^{\top}(\tilde{\sigma}(u) \nu)$ instead of $(\sigma(u) \nu)$ and $s$ instead of $\dot{d}$. This motivates the introduction of a different Lagrange multiplier $\lambda$ that represents $-R_{\theta}^{\top}(\tilde{\sigma}(u) \nu)$,

$$
\lambda:=R_{\theta}^{\top} \tilde{\lambda}
$$

and we derive $b(\eta, \tilde{\lambda})=b\left(R_{\theta}^{\top} \eta, \lambda\right)$.

We are now in the position to gather the new representation of the inertial term (2.17), the contact (2.19) and friction conditions (2.20), and obtain the following weak mixed formulation: Find $(u, \lambda)$ with $u(t) \in \mathcal{V}, \lambda(t) \in \mathcal{M}(\lambda(t))$ such that

$$
\begin{aligned}
m\left(\dot{s}+\dot{\theta} \Pi s, R_{\theta}^{\top} \eta\right)+a_{n l}\left(u, R_{\theta}^{\top} \eta\right)+b\left(R_{\theta}^{\top} \eta, \lambda\right) & =F(\eta), & & \eta \in \mathcal{V}, \\
b_{\nu_{0}}(u, \mu-\lambda)+b_{\tau_{0}}(s, \mu-\lambda) & \leq\left\langle\gamma_{C, \theta}, \mu_{\nu_{0}}-\lambda_{\nu_{0}}\right\rangle, & & \mu \in \mathcal{M}(\lambda) .
\end{aligned}
$$

Considering that, in the above formulation, $\eta$ stands for any variation $\delta \varphi=$ $\delta \theta \Pi R_{\theta} r+R_{\theta} \delta u$ of $\varphi=R(x+u)$, variations with respect to $u$ lead to the choice $\eta=R_{\theta} v$ for any variation $v \in \mathcal{X}$ of $u \in \mathcal{X}$, where $\mathcal{X}$ is the space defined in (2.10). The first line of $(2.22)$ then reads

$$
m(\dot{s}+\dot{\theta} \Pi s, v)+a_{n l}(u, v)+b(v, \lambda)=F_{\theta}(v), \quad v \in \mathcal{X},
$$

where $F_{\theta}(v):=F\left(R_{\theta} v\right)$. Variations with respect to $\theta$ lead to the choice $\eta=\Pi R r$, with $r$ defined in (2.16); hence

$$
m(\dot{s}+\dot{\theta} \Pi s, \Pi r)=F_{\theta}(\Pi r)-b(\Pi r, \lambda) .
$$

Altogether, the weak formulation reads as follows: Find $(u, \lambda, \theta)$, with $u(t) \in \mathcal{X}$, $\lambda(t) \in \mathcal{M}(\lambda(t)), \theta(t) \in \mathbb{R}$ such that

$$
\begin{aligned}
m(\dot{s}+\dot{\theta} \Pi s, \Pi r) & =F_{\theta}(\Pi r)-b(\Pi r, \lambda), & & \\
m(\dot{s}+\dot{\theta} \Pi s, v)+a_{n l}(u, v)+b(v, \lambda) & =F_{\theta}(v), & & v \in \mathcal{X}, \\
b_{\nu_{0}}(u, \mu-\lambda)+b_{\tau_{0}}(s, \mu-\lambda) & \leq\left\langle\gamma_{C, \theta}, \mu_{\nu_{0}}-\lambda_{\nu_{0}}\right\rangle, & & \mu \in \mathcal{M}(\lambda) .
\end{aligned}
$$

Moreover, taking, respectively, into account the linearizations (2.7) and (2.14), we get

$$
a_{n l}\left(u, R_{\theta}^{\top} \eta\right) \approx a\left(u, R_{\theta}^{\top} \eta\right),
$$

where $a(u, \eta):=\int_{\Omega}(\sigma(u): \varepsilon(\eta))$ with $(\sigma(u))_{i j}:=C_{i j m l}(\varepsilon(u))_{m l}$. Here $\left(C_{i j m l}\right)$ denotes the elasticity tensor of the Saint Venant-Kirchhoff material law. Using this linearization in (2.25), we formulate our first corotational model: Find $(u, \lambda, \theta)$, with $u(t) \in \mathcal{X}$, $\lambda(t) \in \mathcal{M}(\lambda(t)), \theta(t) \in \mathbb{R}$ such that

$$
\begin{aligned}
m(\dot{s}+\dot{\theta} \Pi s, \Pi r) & =F_{\theta}(\Pi r)-b(\Pi r, \lambda), & & \\
m(\dot{s}+\dot{\theta} \Pi s, v)+a(u, v)+b(v, \lambda) & =F_{\theta}(v), & & v \in \mathcal{X}, \\
b_{\nu_{0}}(u, \mu-\lambda)+b_{\tau_{0}}(s, \mu-\lambda) & \leq\left\langle\gamma_{C, \theta}, \mu_{\nu_{0}}-\lambda_{\nu_{0}}\right\rangle, & & \mu \in \mathcal{M}(\lambda) .
\end{aligned}
$$

Copyright $@$ by SIAM. Unauthorized reproduction of this article is prohibited. 
Observe that, in the above formulation, the elastic equilibrium equation is linear in $u$. Therefore, the Lagrange multiplier $\lambda$ represents a linearized contact stress. Thanks to the described approach, the only nonlinearities remain in the scalar unknown $\theta$ and the frictional contact inequality condition. It's crucial to note that performing the proposed linearization after the splitting of the deformation into $\theta$ and $u$ gives quite a different model than the standard linearization. In fact, a formal testing of the second line of (2.27) with $v=\Pi r$ would result in a nonvanishing elastic work $a(u, \Pi r) \neq 0$ in general. This is a well-known drawback of the standard linear elastic models: Zeroenergy modes are not rigid body motions (cf. [4]). This is remedied by our corotational approach.

2.3. Energy and angular momentum consistency. To obtain an energyconsistent algorithm, we use a linearized version of the persistency condition (2.21), which implies that

$$
b_{\nu_{0}}(s, \lambda)=0 .
$$

Then, multiplying the first line of (2.27) with $\dot{\theta}$, choosing $v=\dot{u} \in \mathcal{X}$ in the second line, and adding the two equations, we obtain the energy-consistency of our approach.

Lemma 2.1. The solution $(u, \lambda, \theta)$ of (2.27) satisfies

$$
\dot{\mathcal{E}}=F_{\theta}(s)-b_{\tau_{0}}(s, \lambda),
$$

where the energy $\mathcal{E}$ is defined by

$$
\mathcal{E}:=\frac{1}{2}\left(\int_{\Omega} \rho|s|^{2}+\int_{\Omega} \sigma(u): \varepsilon(u)\right)=\frac{1}{2}(m(s, s)+a(u, u)) .
$$

Moreover, from the definition of $r$ and $s$, we find that $\dot{r}=\dot{u}$ and, using the skew-symmetry of $\Pi$, we conclude that $0=m(\Pi s, s)=m(\Pi s, \dot{r}+\dot{\theta} \Pi r)$. This gives $m(\dot{s}+\dot{\theta} \Pi s, \Pi r)=m(\dot{s}, \Pi r)-m(\Pi s, \dot{r})=m(\dot{s}, \Pi r)+m(s, \Pi \dot{r})$, which results in the following lemma.

LEMMA 2.2. Condition (2.24) is equivalent to the consistency of the angular momentum

$$
\dot{\mathcal{J}}=F_{\theta}(\Pi r)-b(\Pi r, \lambda),
$$

where the angular momentum $\mathcal{J}$ is defined by

$$
\mathcal{J}:=\int_{\Omega} \rho s \cdot \Pi r=m(s, \Pi r) .
$$

We will adapt the same techniques to show the energy and angular momentum consistency of the time-discretized scheme.

2.4. Linearization of the inertial term. For a small local displacement $u$, $u \ll x$, the approximation $x+u \approx x$ may be used. Since in our corotational model the deformation $u$ also incorporates translations, we therefore approximate $u$ by the mean translation (2.11), i.e.,

$$
r(x, t)=x+u(x, t) \approx x+T(u(x, t))=: \tilde{r} .
$$

Replacing $r$ by $\tilde{r}$ in (2.15), we obtain the simplified form

$$
s(x, t) \approx \tilde{s}(x, t)=\dot{u}(x, t)+\dot{\theta}(t) \Pi(x+T(u(x, t))) .
$$

Copyright (c) by SIAM. Unauthorized reproduction of this article is prohibited. 
Using this approximation, the first two lines of (2.27) read

$$
\begin{aligned}
m(\dot{\tilde{s}}+\dot{\theta} \Pi \tilde{s}, \Pi \tilde{r}) & =F_{\theta}(\Pi \tilde{r})-b(\Pi \tilde{r}, \lambda), \\
m(\dot{\tilde{s}}+\dot{\theta} \Pi \tilde{s}, v)+a(u, v)+b(v, \lambda) & =F_{\theta}(v), \quad v \in \mathcal{X} .
\end{aligned}
$$

The advantage of this linearization consists in the fact that the linearized rotation $\Pi \tilde{r}$ does not have any influence on the elastic term; using the skew-symmetry of $\Pi$, a straightforward computation yields $a(u, \Pi \tilde{r})=a(u, \Pi x)=0$, and hence the two above equations can be combined into one equation using the unconstrained test space $\mathcal{V}$. We use condition (2.9) as a constraint to determine the correct value of $\theta$. The weak formulation of our second corotational model thus reads: Find $(u, \lambda, \theta)$, with $u(t) \in \mathcal{V}$, $\lambda(t) \in \mathcal{M}(\lambda(t)), \theta(t) \in \mathbb{R}$ such that

$$
\begin{aligned}
m(u, \Pi x) & =0, & & \\
m(\dot{\tilde{s}}+\dot{\theta} \Pi \tilde{s}, v)+a(u, v)+b(v, \lambda) & =F_{\theta}(v), & & v \in \mathcal{V}, \\
b_{\nu_{0}}(u, \mu-\lambda)+b_{\tau_{0}}(\tilde{s}, \mu-\lambda) & \leq\left\langle\gamma_{C, \theta}, \mu_{\nu_{0}}-\lambda_{\nu_{0}}\right\rangle, & & \mu \in \mathcal{M}(\lambda) .
\end{aligned}
$$

The energy consistency of the new scheme with linearized $r$ follows analogously to Lemma 2.1 where the simplified energy is defined by

$$
\tilde{\mathcal{E}}:=\frac{1}{2}\left(\int_{\Omega} \rho|\tilde{s}|^{2}+\int_{\Omega} \sigma(u): \varepsilon(u)\right)=\frac{1}{2}(m(\tilde{s}, \tilde{s})+a(u, u)) .
$$

However, the angular momentum consistency does not hold for this approach. Indeed, let us test the first equation of (2.31) with $v=\Pi \tilde{r}$, keeping in mind the fact that

$$
0=m(\Pi \tilde{s}, \tilde{s})=m(\Pi \tilde{s}, \dot{u}+\dot{\theta} \Pi \tilde{r}) .
$$

The inertial term reduces to

$$
m(\dot{\tilde{s}}+\dot{\theta} \Pi \tilde{s}, \Pi \tilde{r})=m(\dot{\tilde{s}}, \Pi \tilde{r})+m(\tilde{s}, \Pi \dot{u})=\dot{\tilde{J}}+E .
$$

We have denoted by $\tilde{J}=m(\tilde{s}, \Pi \tilde{r})$ the expected angular momentum, and by $E=$ $m(\tilde{s}, \Pi(\dot{u}-\dot{\tilde{r}}))$ the angular momentum consistency error. Using $\dot{\tilde{r}}=T(\dot{u})$, the definition of $\tilde{s}$, the skew-symmetry of $\Pi$, and property (2.12) of $T$, a simple calculation shows that the local consistency error is given by

$$
E=\dot{\theta} m(\Pi x, \Pi(\dot{u}-T(\dot{u})))
$$

which is, in general, not zero but remains small for small variations of velocities.

3. Time-discretized conservative schemes. In this section, we present an energy- and angular-momentum consistent time-discretization of the previous models. We first introduce some notation.

3.1. Midpoint time-discretization. Let us denote by $n$ the time index. We use a midpoint time-discretization which has the property

$$
\dot{a}^{n+\frac{1}{2}} b^{n+\frac{1}{2}}+a^{n+\frac{1}{2}} \dot{b}^{n+\frac{1}{2}}=[\dot{a} b]^{n+\frac{1}{2}},
$$

Copyright $@$ by SIAM. Unauthorized reproduction of this article is prohibited. 
where we have used the following notations:

$$
\star^{n+\frac{1}{2}}:=\frac{\star^{n+1}+\star^{n}}{2}, \star^{n+\frac{1}{2}}:=\frac{\star^{n+1}-\star^{n}}{\Delta t},
$$

with $\Delta t$ the time step and $\star$ a generic time-discretized quantity. The right-hand side $F_{\theta}$ is discretized at time $\left(n+\frac{1}{2}\right) \Delta t$ by

$$
F^{n+\frac{1}{2}}(v):=\int_{\Omega} f^{n+\frac{1}{2}} \cdot R_{\theta^{n+\frac{1}{2}}} v+\int_{\Gamma} g^{n+\frac{1}{2}} \cdot R_{\theta^{n+\frac{1}{2}}} v .
$$

3.2. Algorithm. We propose to discretize the two first equations in (2.27) at time $\left(n+\frac{1}{2}\right) \Delta t$ as follows. Assuming $u^{n} \in \mathcal{X}, s^{n}$ and $\theta^{n} \in \mathbb{R}$ are given, we look for $u^{n+1} \in \mathcal{X}$ and $\theta^{n+1} \in \mathbb{R}$ such that

$$
\begin{gathered}
m\left(\dot{s}^{n+\frac{1}{2}}+\dot{\theta}^{n+\frac{1}{2}} \Pi s^{n+\frac{1}{2}}, \Pi r^{n+\frac{1}{2}}\right)+b\left(\Pi r^{n+\frac{1}{2}}, \lambda^{n+\frac{1}{2}}\right)=F^{n+\frac{1}{2}}\left(\Pi r^{n+\frac{1}{2}}\right), \\
m\left(\dot{s}^{n+\frac{1}{2}}+\dot{\theta}^{n+\frac{1}{2}} \Pi s^{n+\frac{1}{2}}, v\right)+a\left(u^{n+\frac{1}{2}}, v\right)+b\left(v, \lambda^{n+\frac{1}{2}}\right)=F^{n+\frac{1}{2}}(v) \quad v \in \mathcal{X}
\end{gathered}
$$

complemented by the relation

$$
s^{n+\frac{1}{2}}=\dot{u}^{n+\frac{1}{2}}+\dot{\theta}^{n+\frac{1}{2}} \Pi r^{n+\frac{1}{2}} .
$$

The time-discretization of the contact inequality (third line of $(2.27)$ ) is, however, more involved. As mentioned in section 2.1, it is not possible to fulfill the contact conditions (2.19) and the persistency condition (2.21) simultaneously. To define an energy-consistent time discretization, we follow the approach by Laursen and Chawla $[3,17]$. The contact condition $(2.19)$ is replaced by a discrete condition depending only on $s^{n+\frac{1}{2}}$. For the linearized elastic term, this condition reads

$$
\begin{aligned}
& \gamma_{C, \theta}^{n}>0 \Rightarrow \sigma_{\nu_{0}}\left(u^{n+\frac{1}{2}}\right)=0, \\
& \gamma_{C, \theta}^{n} \leq 0 \Rightarrow\left(s_{\nu_{0}}^{n+\frac{1}{2}} \leq 0, \quad \sigma_{\nu_{0}}\left(u^{n+\frac{1}{2}}\right) \leq 0, \quad \sigma_{\nu_{0}}\left(u^{n+\frac{1}{2}}\right) s_{\nu_{0}}^{n+\frac{1}{2}}=0\right),
\end{aligned}
$$

where $\gamma_{C, \theta}^{n}:=\gamma_{C, \theta^{n}}$. By this modification, a small penetration (of order $s \Delta t$ ) is permitted. On the other hand, it can be easily verified that a discrete persistency condition

$$
b_{\nu_{0}}\left(s^{n+\frac{1}{2}}, \lambda^{n+\frac{1}{2}}\right)=0
$$

holds for this modified condition. Condition (3.5) is equivalent to

$$
s_{\nu_{0}}^{n+\frac{1}{2}} \leq \hat{\gamma}_{C, \theta}^{n}, \quad \sigma_{\nu_{0}}\left(u^{n+\frac{1}{2}}\right) \leq 0, \quad \sigma_{\nu_{0}}\left(u^{n+\frac{1}{2}}\right)\left(s_{\nu_{0}}^{n+\frac{1}{2}}-\hat{\gamma}_{C, \theta}^{n}\right)=0,
$$

with

$$
\hat{\gamma}_{C, \theta}^{n}:= \begin{cases}\infty & \gamma_{C, \theta}^{n}>0 \\ 0 & \gamma_{C, \theta}^{n} \leq 0\end{cases}
$$

In combination with the friction condition, we arrive at the following discretization of the contact inequality:

$$
b\left(s^{n+\frac{1}{2}}, \mu-\lambda^{n+\frac{1}{2}}\right) \leq\left\langle\hat{\gamma}_{C, \theta}^{n}, \mu_{\nu_{0}}-\lambda_{\nu_{0}}^{n+\frac{1}{2}}\right\rangle, \quad \mu \in \mathcal{M}\left(\lambda^{n+\frac{1}{2}}\right) .
$$

Observe that the contact condition is now exclusively written in terms of $s^{n+\frac{1}{2}}$. 
Hence we have the following algorithm for our first corotational formulation: Given $\theta_{0}, u_{0}, s_{0}$, the external forces $\left(f^{n+\frac{1}{2}}\right)^{n \in \mathbb{N}}$ and $\left(g^{n+\frac{1}{2}}\right)^{n \in \mathbb{N}}$, suppose that $\theta^{n} \in \mathbb{R}$, $u^{n} \in \mathcal{X}$, and $s^{n}$ have already been computed. Then, the computation of $\theta^{n+1} \in \mathbb{R}$, $u^{n+1} \in \mathcal{X}, s^{n+1} \in \mathcal{V}$, and $\lambda^{n+\frac{1}{2}} \in \mathcal{M}\left(\lambda^{n+\frac{1}{2}}\right)$ is carried out by solving

$$
\begin{gathered}
m\left(\dot{s}^{n+\frac{1}{2}}+\dot{\theta}^{n+\frac{1}{2}} \Pi s^{n+\frac{1}{2}}, \Pi r^{n+\frac{1}{2}}\right)+b\left(\Pi r^{n+\frac{1}{2}}, \lambda^{n+\frac{1}{2}}\right)=F^{n+\frac{1}{2}}\left(\Pi r^{n+\frac{1}{2}}\right), \\
m\left(\dot{s}^{n+\frac{1}{2}}+\dot{\theta}^{n+\frac{1}{2}} \Pi s^{n+\frac{1}{2}}, v\right)+a\left(u^{n+\frac{1}{2}}, v\right)+b\left(v, \lambda^{n+\frac{1}{2}}\right)=F^{n+\frac{1}{2}}(v), \quad v \in \mathcal{X}, \\
b\left(s^{n+\frac{1}{2}}, \mu-\lambda^{n+\frac{1}{2}}\right) \leq\left\langle\hat{\gamma}_{C, \theta}^{n}, \mu_{\nu_{0}}-\lambda_{\nu_{0}}^{n+\frac{1}{2}}\right\rangle, \quad \mu \in \mathcal{M}\left(\lambda^{n+\frac{1}{2}}\right)
\end{gathered}
$$

under the additional kinematical constraint (3.4).

As in the continuous setting, we get the energy consistency by multiplying (3.2) by $\dot{\theta}^{n+\frac{1}{2}}$, testing (3.3) with $\dot{u}^{n+\frac{1}{2}}$, adding the two resulting equations, and using a property (3.1) of the midpoint scheme. We employ the discrete persistency condition (3.6) and find

$$
\mathcal{E}^{n+1}-\mathcal{E}^{n}=\Delta t\left(F^{n+\frac{1}{2}}\left(s^{n+\frac{1}{2}}\right)-b_{\tau_{0}}\left(s^{n+\frac{1}{2}}, \lambda^{n+\frac{1}{2}}\right)\right),
$$

where the discrete energy is defined by

$$
\mathcal{E}^{n}:=\frac{1}{2}\left(m\left(s^{n}, s^{n}\right)+a\left(u^{n}, u^{n}\right)\right) .
$$

By means of the time-discretization of $s^{n+\frac{1}{2}}$, we find

$$
\dot{r}^{n+\frac{1}{2}}=\dot{u}^{n+\frac{1}{2}}=s^{n+\frac{1}{2}}-\dot{\theta}^{n+\frac{1}{2}} \Pi r^{n+\frac{1}{2}},
$$

and using (3.1), we conclude that (3.2) is equivalent to

$$
\mathcal{J}^{n+1}-\mathcal{J}^{n}=\Delta t\left(F^{n+\frac{1}{2}}\left(\Pi r^{n+\frac{1}{2}}\right)-b\left(\Pi r^{n+\frac{1}{2}}, \lambda^{n+\frac{1}{2}}\right)\right),
$$

where the discrete angular momentum is defined by:

$$
\mathcal{J}^{n}:=m\left(s^{n}, \Pi r^{n}\right)
$$

For the second formulation (linearized $r$ ), we do not have to work in the space $\mathcal{X}$ but can use equation (2.9) as a constraint. We get the following algorithm: Given $\theta_{0}, u_{0}, s_{0}$, the external forces $\left(f^{n+\frac{1}{2}}\right)^{n \in \mathbb{N}}$ and $\left(g^{n+\frac{1}{2}}\right)^{n \in \mathbb{N}}$, suppose that $\theta^{n}, u^{n} \in \mathcal{X}$, and $s^{n}$ have already been computed. Then, the computation of $\theta^{n+1} \in \mathbb{R}, u^{n+1} \in \mathcal{V}$, $\tilde{s}^{n+1} \in \mathcal{V}$, and $\lambda^{n+\frac{1}{2}} \in \mathcal{M}\left(\lambda^{n+\frac{1}{2}}\right)$ is carried out by solving,

$$
\begin{gathered}
m\left(u^{n+\frac{1}{2}}, \Pi x\right)=0, \\
m\left(\dot{\tilde{s}}^{n+\frac{1}{2}}+\dot{\theta}^{n+\frac{1}{2}} \Pi \tilde{s}^{n+\frac{1}{2}}, v\right)+a\left(u^{n+\frac{1}{2}}, v\right)+b\left(v, \lambda^{n+\frac{1}{2}}\right)=F^{n+\frac{1}{2}}(v) \quad v \in \mathcal{V}, \\
b\left(\tilde{s}^{n+\frac{1}{2}}, \mu-\lambda^{n+\frac{1}{2}}\right) \leq\left\langle\hat{\gamma}_{C, \theta}^{n}, \mu_{\nu_{0}}-\lambda_{\nu_{0}}^{n+\frac{1}{2}}\right\rangle, \quad \mu \in \mathcal{M}\left(\lambda^{n+\frac{1}{2}}\right)
\end{gathered}
$$

under the constraint

$$
\tilde{s}^{n+\frac{1}{2}}=\dot{u}^{n+\frac{1}{2}}+\dot{\theta}^{n+\frac{1}{2}} \Pi \tilde{r}^{n+\frac{1}{2}} .
$$

Establishing the corresponding discrete energy evolution mimics the proof from the continuous setting.

Copyright $@$ by SIAM. Unauthorized reproduction of this article is prohibited. 
4. Space discretization. In this section, we present a suitable space discretization for the time-discretized systems. Standard finite elements are used to provide an approximation of $\mathcal{V}$, but the construction of a suitable space-discretization of the space $\mathcal{X}$ is more involved. The domain $\Omega$ is assumed to be exactly represented by a mesh consisting of quadrilaterals and/or triangles in the two-dimensional setting and hexahedra and/or tetrahedra in the $3 D$. We use a conforming finite element space of lowest order and denote by $\phi_{i}$ the nodal vector basis functions; they span the finite element space $\mathcal{V}_{h}$. The mass matrix is denoted by $M, M_{i j}:=m\left(\phi_{i}, \phi_{j}\right)$, and the stiffness matrix for the linearized material law by $S, S_{i j}:=a\left(\phi_{i}, \phi_{j}\right)$. The identity matrix is denoted by $I$.

Applying this discretization to the second corotational scheme (with linearized $r$ ), the first two lines of (3.10) read

$$
\begin{aligned}
M u_{h}^{n+\frac{1}{2}} \cdot \Pi x & =0, \\
M\left(\dot{\tilde{s}}_{h}^{n+\frac{1}{2}}+\dot{\theta}^{n+\frac{1}{2}} \Pi \tilde{s}_{h}^{n+\frac{1}{2}}\right)+S u_{h}^{n+\frac{1}{2}}+B \lambda_{h}^{n+\frac{1}{2}} & =f_{h}^{n+\frac{1}{2}},
\end{aligned}
$$

with $\left(f_{h}^{n+\frac{1}{2}}\right)_{i}:=F^{n+\frac{1}{2}}\left(\phi_{i}\right)$, and $x$ is the vector whose entries are the vertex coordinates.

For the space-discretization of $\mathcal{M}$, we use the dual Lagrange multipliers space $\mathcal{M}_{h}$ [26], which is given in terms of the dual basis functions $\psi_{i}$. By the definition of the dual basis, the coupling matrix $B$ defined by $B_{i j}:=b\left(\phi_{i}, \psi_{j}\right)$ is then a diagonal matrix, and the weak inequality constraint (3.8) yields a nodewise inequality. More details and a suitable discretization of the convex set $\mathcal{M}\left(\lambda_{h}\right)$ can be found in $[14,15]$.

4.1. Finite element approximation of the space $\mathcal{X}$. As condition (2.9) is not fulfilled by standard finite element basis functions, we use a Lagrange multiplier approach for our first corotational scheme to guarantee (2.9) and state the problem in a saddle-point framework; see [24].

We define the subspace $\mathcal{X}_{h}$ of $\mathcal{X}$ by $\mathcal{X}_{h}:=\mathcal{X} \cap \mathcal{V}_{h}$. To guarantee that $u \in \mathcal{V}_{h}$ is also in $\mathcal{X}_{h}$, i.e., satisfies (2.9), we introduce a Lagrange multiplier $\alpha \in \mathbb{R}$. Equation (3.3) then reads at the fully discretized level:

$$
\begin{aligned}
M\left(\dot{s}_{h}^{n+\frac{1}{2}}+\dot{\theta}^{n+\frac{1}{2}} \Pi s_{h}^{n+\frac{1}{2}}\right)+S u_{h}^{n+\frac{1}{2}}+B \lambda_{h}^{n+\frac{1}{2}}+\alpha^{n+\frac{1}{2}} M \Pi x & =f_{h}^{n+\frac{1}{2}} \\
M u_{h}^{n+\frac{1}{2}} \cdot \Pi x & =0 \\
\left(M\left(\dot{s}_{h}^{n+\frac{1}{2}}+\dot{\theta}^{n+\frac{1}{2}} \Pi s_{h}^{n+\frac{1}{2}}\right)+B \lambda_{h}^{n+\frac{1}{2}}\right) \cdot \Pi r_{h}^{n+\frac{1}{2}} & =f_{h}^{n+\frac{1}{2}} \cdot \Pi r_{h}^{n+\frac{1}{2}}
\end{aligned}
$$

This equation system is completed using the contact constraints. Observe that we now use two Lagrange multipliers, namely, $\lambda_{h}$ representing the contact stress and $\alpha$ to ensure that $u_{h}$ is in the correct space.

4.2. Equation systems. We recall that the contact and friction conditions are given only in terms of $s$. Therefore it is convenient to reformulate the equations with respect to the unknown $s_{h}^{n+\frac{1}{2}}$. We start with the first approach, $r=x+u$. From the relationship

$$
s_{h}^{n+\frac{1}{2}}=\frac{2}{\Delta t}\left(u_{h}^{n+\frac{1}{2}}-u_{h}^{n}\right)+\dot{\theta}^{n+\frac{1}{2}} \Pi\left(x+u_{h}^{n+\frac{1}{2}}\right),
$$


we deduce that

$$
u_{h}^{n+\frac{1}{2}}=\left(\frac{2}{\Delta t} I+\dot{\theta}^{n+\frac{1}{2}} \Pi\right)^{-1}\left(s_{h}^{n+\frac{1}{2}}+\frac{2}{\Delta t} u_{h}^{n}-\dot{\theta}^{n+\frac{1}{2}} \Pi x\right) .
$$

Plugging this into (4.2)-(4.4) and using that $u_{h}^{n} \in \mathcal{X}_{h}$, gives the following system:

$$
\begin{aligned}
A s_{h}^{n+\frac{1}{2}}+B \lambda_{h}^{n+\frac{1}{2}}+\alpha M \Pi x & =f_{\mathrm{rhs}}, \\
q_{x} \cdot s_{h}^{n+\frac{1}{2}} & =q_{\mathrm{rhs}}, \\
g\left(\dot{\theta}^{n+\frac{1}{2}}\right) & =0,
\end{aligned}
$$

with

$$
A:=M\left(\frac{2}{\Delta t} I+\dot{\theta}^{n+\frac{1}{2}} \Pi\right)+S C_{n}, \quad C_{n}:=\left(\frac{2}{\Delta t} I+\dot{\theta}^{n+\frac{1}{2}} \Pi\right)^{-1} .
$$

The right-hand side for the first equation is given by

$$
f_{\mathrm{rhs}}:=f_{h}^{n+\frac{1}{2}}+\frac{2}{\Delta t} M s_{h}^{n}-\frac{2}{\Delta t} S C_{n} u_{h}^{n}+\dot{\theta}^{n+\frac{1}{2}} S C_{n} \Pi x ;
$$

for the second equation, we have

$$
q_{x}:=C_{n}^{\top} M \Pi x, \quad q_{\mathrm{rhs}}:=\dot{\theta}^{n+\frac{1}{2}}\left(M C_{n} \Pi x\right) \cdot \Pi x-\frac{2}{\Delta t} M C_{n} u_{h}^{n} \cdot \Pi x .
$$

For the third equation, we use

$$
g(\dot{\theta}):=M\left(\frac{2}{\Delta t}\left(s_{h}^{n+\frac{1}{2}}(\dot{\theta})-s_{h}^{n}\right)+\dot{\theta} \Pi s_{h}^{n+\frac{1}{2}}(\dot{\theta})+B \lambda_{h}^{n+\frac{1}{2}}(\dot{\theta})-f_{h}^{n+\frac{1}{2}}(\dot{\theta})\right) \cdot \Pi r_{h}^{n+\frac{1}{2}}(\dot{\theta}) .
$$

For the second approach, $\tilde{r}=x+T(u)$, the situation is much simpler. As noted before, we can work in the space $\mathcal{V}_{h}$, and thus no Lagrange multiplier $\alpha$ is needed. Moreover, the representation of $u_{h}^{n+\frac{1}{2}}$ in terms of $s_{h}^{n+\frac{1}{2}}$ (and $T\left(u_{h}^{n+\frac{1}{2}}\right)$ ) has the simpler form

$$
u_{h}^{n+\frac{1}{2}}=\frac{\Delta t}{2} s_{h}^{n+\frac{1}{2}}-\frac{\Delta t \dot{\theta}^{n+\frac{1}{2}}}{2} \Pi\left(x+T\left(u_{h}^{n+\frac{1}{2}}\right)\right)+u_{h}^{n}
$$

We exploit the fact that $\Pi$ is skew-symmetric, and $T\left(u_{h}^{n+\frac{1}{2}}\right)$ is a translation. Thus, $S \Pi\left(x+T\left(u_{h}^{n+\frac{1}{2}}\right)\right)=0$, and we finally get the following system

$$
\begin{gathered}
A s_{h}^{n+\frac{1}{2}}+B \lambda_{h}^{n+\frac{1}{2}}=f_{\mathrm{rhs}} \\
g\left(\dot{\theta}^{n+\frac{1}{2}}\right)=0,
\end{gathered}
$$

with

$$
A:=M\left(\frac{2}{\Delta t} I+\dot{\theta}^{n+\frac{1}{2}} \Pi\right)+\frac{\Delta t}{2} S, \quad f_{\mathrm{rhs}}:=f_{h}^{n+\frac{1}{2}}+\frac{2}{\Delta t} M s_{h}^{n}-S u_{h}^{n},
$$

Copyright $@$ ㅇ by SIAM. Unauthorized reproduction of this article is prohibited. 
and

$$
g(\dot{\theta}):=M u_{h}^{n+\frac{1}{2}}(\dot{\theta}) \cdot \Pi x
$$

Knowing $s_{h}^{n+\frac{1}{2}}$, integration yields

$$
T\left(s_{h}^{n+\frac{1}{2}}\right)=\left(\frac{2}{\Delta t} I+\dot{\theta}^{n+\frac{1}{2}} \Pi\right) T\left(u_{h}^{n+\frac{1}{2}}\right)-\frac{2}{\Delta t} T\left(u_{h}^{n}\right),
$$

and therefore

$$
T\left(u_{h}^{n+\frac{1}{2}}\right)=C_{n}\left(T\left(s_{h}^{n+\frac{1}{2}}\right)+\frac{2}{\Delta t} T\left(u_{h}^{n}\right)\right) .
$$

Using this value, $u_{h}^{n+\frac{1}{2}}$ is computed by (4.5).

5. Solving the nonlinearities. The fully discretized systems are linear in $s$, but the nonlinearity in $\theta$ and the contact nonlinearities remain. In this section, we give an algorithm to solve these nonlinearities. Our algorithm consists of an outer loop to determine the correct contact set and an inner-Newton loop to resolve the correct value of $\theta$. Within the inner-Newton loop, a linear equation system depending on the contact set and $\theta$ has to be solved.

To solve the contact constraints, we use a primal-dual active set strategy [15]. The adaption to our problem setting is quite straightforward as we are in the case of a standard frictional contact problem, where only the acceleration term and the right-hand side is modified. We remark that the application of the active set strategy can be interpreted as a semismooth-Newton scheme [13]. In this section, we outline the approach and refer to [15] for further details.

5.1. Primal-dual active set strategy. To solve the friction and contact inequalities (2.20), (3.5), respectively, the set of nodes on $\Gamma_{C}$ is divided into inactive nodes, sticky nodes, and slippy nodes. The pointwise contact conditions then give rise to a specific boundary condition for each set of nodes and thus yields a linear equation system to solve. Knowing the solution of this linear system, the set of inactive, sticky, and slippy nodes is updated. We note that there are different possibilities to derive the boundary conditions and to determine the contact sets, especially in the frictional case. This choice has an influence on the convergence rate of the active set strategy. To simplify the notation herein, we restrict ourselves to the case of frictionless contact and refer to [14] for details about the sets and boundary conditions to be used in the frictional case.

For frictionless contact, the nodes on the contact boundary $\Gamma_{C}$ are divided into the set of active nodes $\mathcal{A}$ and the set of inactive nodes $\mathcal{I}$. The set of inner nodes and nodes on the Neumann boundary is denoted by $\mathcal{N}$. For active nodes, we have homogeneous Dirichlet boundary conditions for $s$ in a normal direction and $\lambda_{p, \tau_{0}}=0$, while for inactive nodes, we impose $\lambda_{p}=0$.

5.2. Matricial representation. Let us now detail the matricial representation of the system to solve at each Newton iteration within one active set prediction. Here, we assume that both the partition into active and inactive nodes as well as the value of $\theta$ is known. We focus on the first approach with $r=x+u$ and remark that the second approach can be treated in the same way with a different matrix $A$, a different right-hand side, and by omitting the terms introduced by the Lagrange multiplier $\alpha$. 
We introduce the matrices $N$ and $T$, which have the normal and tangential vectors $\nu_{0}, \tau_{0}$ of the vertex in the corresponding line, denote the vector $M \Pi x$ by $C$ and the vector $q_{x}^{\top}$ by $Q^{\top}$, and recall that the matrix $D:=B$ is a diagonal matrix. Then, using the contact condition in a normal direction and zero stress in a tangential direction, we arrive at the following system:

$$
\left(\begin{array}{cccccc}
A_{\mathcal{N N}} & A_{\mathcal{N I}} & A_{\mathcal{N A}} & C_{\mathcal{N}} & 0 & 0 \\
A_{\mathcal{I N}} & A_{\mathcal{I I}} & A_{\mathcal{I} \mathcal{A}} & C_{\mathcal{I}} & D_{\mathcal{I}} & 0 \\
A_{\mathcal{A N}} & A_{\mathcal{A I}} & A_{\mathcal{A} \mathcal{A}} & C_{\mathcal{A}} & 0 & D_{\mathcal{A}} \\
Q_{\mathcal{N}} & Q_{\mathcal{I}} & Q_{\mathcal{A}} & 0 & 0 & 0 \\
0 & 0 & N_{\mathcal{A}} & 0 & 0 & 0 \\
0 & 0 & 0 & 0 & 0 & T_{\mathcal{A}} \\
0 & 0 & 0 & 0 & I_{\mathcal{I}} & 0
\end{array}\right)\left(\begin{array}{c}
s_{\mathcal{N}} \\
s_{\mathcal{I}} \\
s_{\mathcal{A}} \\
\alpha \\
\lambda_{\mathcal{I}} \\
\lambda_{\mathcal{A}}
\end{array}\right)=\left(\begin{array}{c}
f_{\mathcal{N}} \\
f_{\mathcal{I}} \\
f_{\mathcal{A}} \\
q \\
0 \\
0 \\
0
\end{array}\right)
$$

Now, using the dual Lagrange multipliers, a static condensation of $\lambda$ (see [15]) leads to

$$
\left(\begin{array}{cccc}
A_{\mathcal{N N}} & A_{\mathcal{N} \mathcal{I}} & A_{\mathcal{N} \mathcal{A}} & C_{\mathcal{N}} \\
A_{\mathcal{I N}} & A_{\mathcal{I I}} & A_{\mathcal{I} \mathcal{A}} & C_{\mathcal{I}} \\
0 & 0 & N_{\mathcal{A}} & 0 \\
T_{\mathcal{A}} A_{\mathcal{A N}} & T_{\mathcal{A}} A_{\mathcal{A I}} & T_{\mathcal{A}} A_{\mathcal{A} \mathcal{A}} & T_{\mathcal{A}} C_{\mathcal{A}} \\
Q_{\mathcal{N}} & Q_{\mathcal{I}} & Q_{\mathcal{A}} & 0
\end{array}\right)\left(\begin{array}{c}
s_{\mathcal{N}} \\
s_{\mathcal{I}} \\
s_{\mathcal{A}} \\
\alpha
\end{array}\right)=\left(\begin{array}{c}
f_{\mathcal{N}} \\
f_{\mathcal{I}} \\
0 \\
T_{\mathcal{A}} f_{\mathcal{A}} \\
q
\end{array}\right)
$$

which can be solved by a standard solver. We remark that the Lagrange multiplier $\lambda$ can be computed a posteriori by

$$
\lambda=D^{-1}\left(f_{\mathcal{S}}-\alpha C_{\mathcal{S}}-A_{\mathcal{S N}} s_{\mathcal{N}}-A_{\mathcal{S} S} s_{\mathcal{S}}\right)
$$

where the set $\mathcal{S}$ is the set of vertices on $\Gamma_{C}, \mathcal{S}:=\mathcal{I} \cup \mathcal{A}$.

5.3. Newton scheme. To determine the correct value of $\theta$, we use a standard Newton scheme

$$
\dot{\theta}_{k+1}^{n+\frac{1}{2}}=\dot{\theta}_{k}^{n+\frac{1}{2}}-\frac{g\left(\dot{\theta}_{k}^{n+\frac{1}{2}}\right)}{g^{\prime}\left(\dot{\theta}_{k}^{n+\frac{1}{2}}\right)} .
$$

Here, $g$ is computed by using the values $s_{h}^{n+\frac{1}{2}}$ and $u_{h}^{n+\frac{1}{2}}$, and $\lambda_{h}^{n+\frac{1}{2}}$ is obtained by solving the linear system (5.1).

To compute the derivative of $g$ with respect to $\dot{\theta}^{n+\frac{1}{2}}$, the derivative of $s_{h}^{n+\frac{1}{2}}$ is needed. Differentiating (5.1) with respect to $\dot{\theta}^{n+\frac{1}{2}}$ gives

$$
\tilde{A} \frac{d s_{h}^{n+\frac{1}{2}}}{d \dot{\theta}}+\frac{d \tilde{A}}{d \dot{\theta}} s_{h}^{n+\frac{1}{2}}=\frac{d \tilde{f}}{d \dot{\theta}},
$$

where $\tilde{A}$ is the system matrix and $\tilde{f}$ is the right-hand side of (5.1). Thus, $\frac{d s_{h}^{n+\frac{1}{2}}}{d \dot{\theta}}$ can be computed by solving system (5.1) with a different right-hand side.

Copyright (c) by SIAM. Unauthorized reproduction of this article is prohibited. 
6. Numerical examples. In this section, we present some two- and threedimensional numerical examples making use of the Saint Venant-Kirchhoff stored energy function. The two-dimensional examples are computed in the plane strain setting orthogonally to the rotation axis.

We use both corotational formulations presented in section 2 and compare them with the standard energy-consistent discretization of the Saint Venant-Kirchhoff model without linearized strains.

6.1. Initial conditions. Let $\dot{\theta}_{0}$ be an initial angular velocity, $\dot{T}_{0}$ an initial translation speed, and $u_{0}$ the initial elastic displacement of the structure. We are interested in such initial conditions rendering the movement steady. More precisely, in the absence of external loading or contact forces, the dynamic solution would be given by the following deformation mapping:

$$
\varphi(x, t)=R_{t \dot{\theta}_{0}}\left(x+u_{0}(x)\right)+t \dot{T}_{0}
$$

In the presence of the first corotational model, this is tantamount to finding $u_{0} \in \mathcal{V}$ such that

$$
\left(\dot{\theta}_{0}\right)^{2} m\left(\Pi^{2}\left(x+u_{0}\right), v\right)+a\left(u_{0}, v\right)=0, \quad v \in \mathcal{V} .
$$

We use the initial velocity $s_{0}=\dot{\theta}_{0} \Pi\left(x+u_{0}\right)+\dot{T}_{0}$. Note the condition $u_{0} \in \mathcal{X}$ is automatically enforced, indeed, using $v=\Pi x$ above proves that $m\left(u_{0}, \Pi x\right)=0$.

For the linearized corotational model, we compute that

$$
\begin{aligned}
& a\left(u_{0}, v\right)=-\left(\dot{\theta}_{0}\right)^{2} m\left(\Pi^{2} x, v\right), \quad v \in \mathcal{V}, \\
& \int_{\Omega} \rho u_{0}=0, \quad m\left(u_{0}, \Pi x\right)=0 .
\end{aligned}
$$

Here the two extra conditions imply uniqueness of the solution. The initial velocity is given by $s_{0}=\dot{\theta}_{0} \Pi x+\dot{T}_{0}$.

For the nonlinear model, the initial deformation $\varphi_{0}$ can be determined up to a rotation. To fix this indetermination, we look for $\varphi_{0} \in \mathcal{X}$ such that

$$
\left(\dot{\theta}_{0}\right)^{2} m\left(\Pi^{2} \varphi_{0}, v\right)+a_{n l}\left(\varphi_{0}-x, v\right)=0, \quad v \in \mathcal{X} .
$$

The initial velocity is then given by $\dot{\varphi}_{0}=\dot{\theta}_{0} \Pi \varphi_{0}+\dot{T}_{0}$.

Observe that all of these initial velocity fields are rigid body motion velocity fields. They slightly differ, because the models vary in terms of the linearization of strains and $r$. It can be easily verified that the time-discretization for the two corotational formulations exactly recover this rigid body motion, whereas the midpoint scheme for the standard nonlinear model does not; see [24].

6.2. A computation of the rotation angle and the translation. In order to compare the three schemes, we compute in a postprocess the rotation and translation of the deformed body. For the corotational schemes, the rotation angle appears as unknown and is therefore a part of the solution. The translation $T_{n}$ is computed by

$$
T_{n}:=R_{\theta_{n}}^{\top} T\left(u_{n}\right)=R_{\theta_{n}}^{\top} \frac{\int_{\Omega} \rho u_{n}}{\int_{\Omega} \rho} .
$$



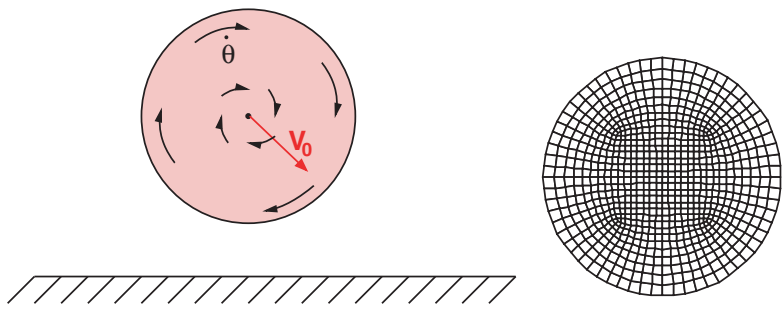

Fig. 6.1. Ball on plane. Left: Problem setting. Right: Grid.
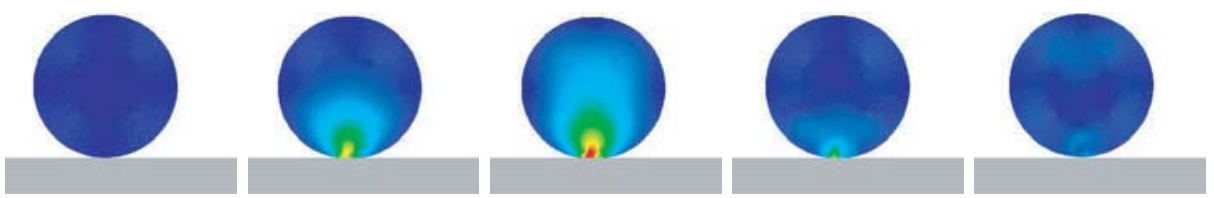

Fig. 6.2. Ball on plane without friction. Deformed body and effective stress at time $t=0.012$, $0.018,0.024,0.03$, and 0.036 .
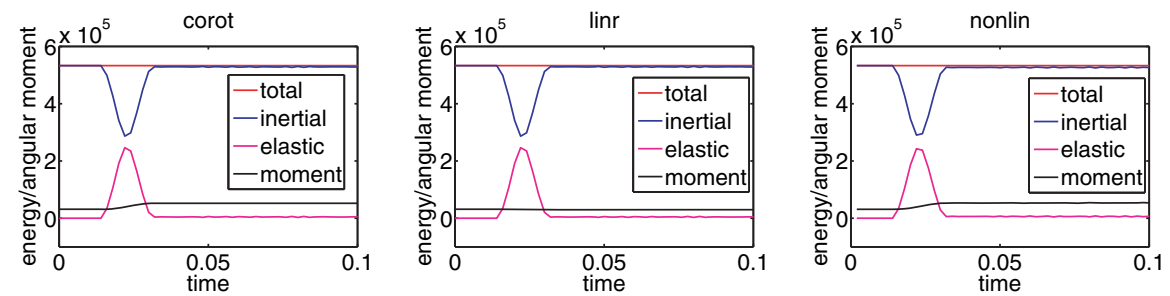

FIG. 6.3. Ball on plane without friction. Energies and angular momentum for corotational (left), linearized $r$ (middle), and standard nonlinear scheme (right).

For the nonlinear scheme, the rotation angle $\theta_{n}$ is computed from the solution $\varphi_{n}$ through the minimization condition (2.9), i.e.,

$$
\int_{\Omega} \rho R_{\theta_{n}}^{\top} \varphi_{n} \cdot \Pi x=0
$$

and the translation $T_{n}$ by

$$
T_{n}:=T\left(\varphi_{n}\right)=\frac{\int_{\Omega} \rho \varphi_{n}}{\int_{\Omega} \rho} .
$$

6.3. Ball on plane. In our first example, we consider a rotating ball impacting a rigid plane; see Figure 6.1. We compare our schemes with the standard nonlinear scheme using a relatively stiff ball and small time steps. The domain $\Omega$ is a ball of radius $r=10$ (see Figure 6.1), and the initial distance between the center of the ball and the plane is 10.5. As material parameters, we use $E=1.62 \cdot 10^{7}, \nu=0.2$, and $\rho=1$. The initial conditions are given by $\dot{\theta}_{0}=2, \dot{T}_{0}=V_{0}=(40,-40)^{\top}$, and as a time step we choose $\Delta t=0.002$.

First, we consider the frictionless contact problem. The solution at various time steps is plotted in Figure 6.2. Figure 6.3 illustrates energy and angular momentum evolutions for the three schemes. All schemes are energy-conserving and yield almost the same energy evolution. Moreover, as predicted by the theory, the nonlinearized 

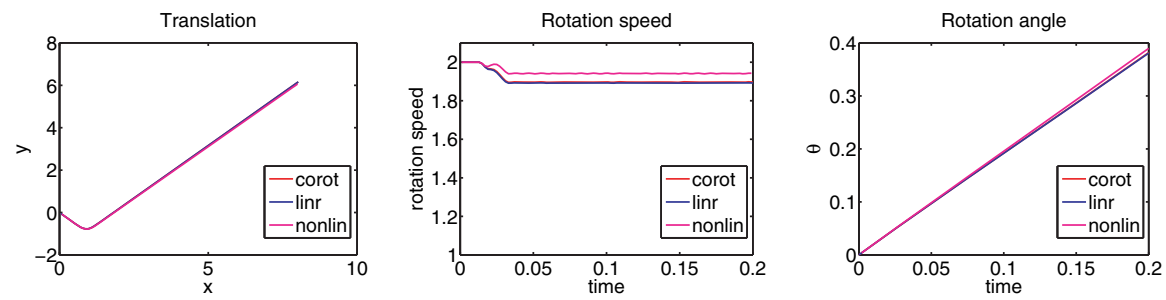

FIG. 6.4. Ball on plane without friction. Translation (left), angular velocity (middle), rotation angle for corotational, linearized $r$ and standard nonlinear scheme (right).
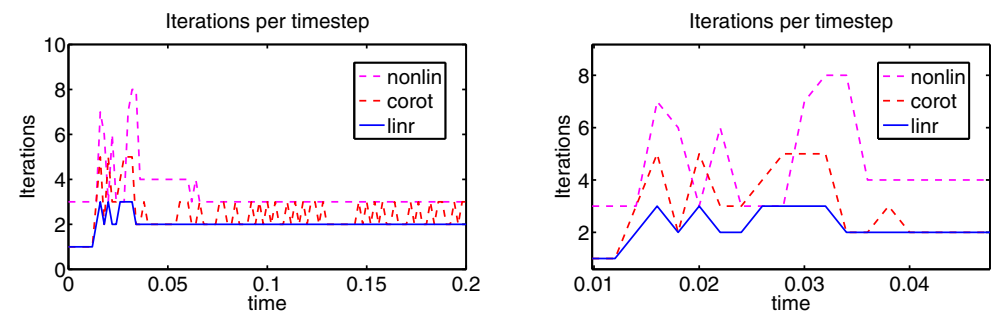

FIG. 6.5. Ball on plane without friction. Comparison of iterations per time step. Right: Interval of contact (zoom).

corotational scheme and the standard nonlinear scheme yield the same angular momentum conservation, unlike the linearized corotational variant.

The translations, angular velocities, and rotation angles are depicted in Figure 6.4. All of the schemes are in good agreement with each other regarding the prediction of the translational component. However, the rotational velocity obtained by the standard nonlinear scheme differs from both corotational predictions. This can be explained by the polygonal shape of the discretized domain. Small changes in the rotation angle therefore suffice to change contact mechanics and lead to different angular velocities after contact. Hence even small changes in the rotation angle lead to a different contact behavior and hence to different rotational speeds after contact. However, as the time step $\Delta t$ goes to zero, all of the approaches tend to predict the same dynamics. The efficiency of the three schemes is compared in Figure 6.5, where the total number of Newton's iterations per time step is plotted. Before contact, the corotational schemes only require one iteration per time step, as compared to three for the standard nonlinear scheme. This is because the constant rigid body motion is exactly resolved by the corotational schemes. During contact, the number of iterations per time step increases but remain smaller for the corotational schemes. In particular, the scheme with linearized $r$ performs much better and barely requires more iterations than an active-set solver for a linear material model. After contact, the corotational schemes require two iterations for most time steps compared to three or four for the standard nonlinear scheme. Altogether, we see that the corotational schemes require many less iterations as compared to the standard nonlinear scheme, while giving comparable results.

Within the same setting, we compute the solutions for a frictional contact problem with Coulomb friction and a friction coefficient $\mathfrak{F}=0.2$. The solution at various time steps is plotted in Figure 6.6. On Figure 6.7, energy evolution is depicted. Again, good agreement on energy evolution can be observed between our two schemes and the standard nonlinear scheme. As far as angular momentum is concerned, the coro- 

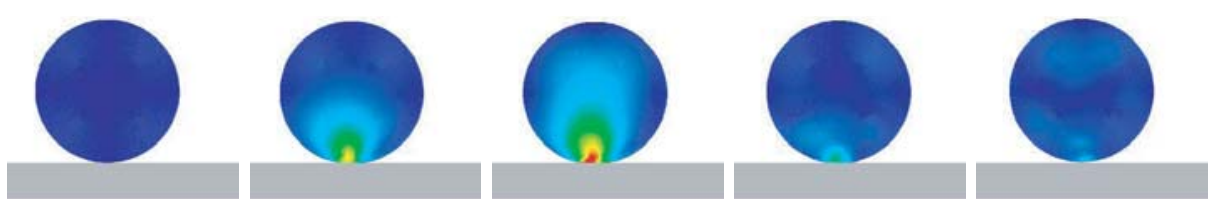

FIG. 6.6. Ball on plane with friction. Deformed body and effective stress at time $t=0.012$, $0.018,0.024,0.03$, and 0.036 .
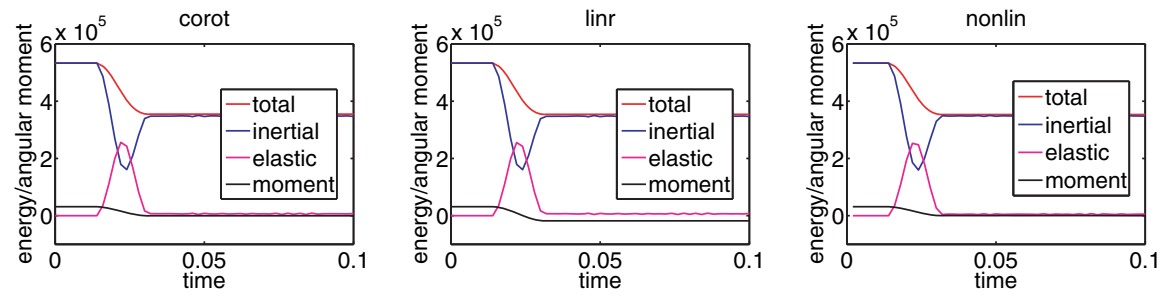

FIG. 6.7. Ball on plane with friction. Energies and angular momentum for corotational (left), linearized $r$ (middle), and standard nonlinear scheme (right).
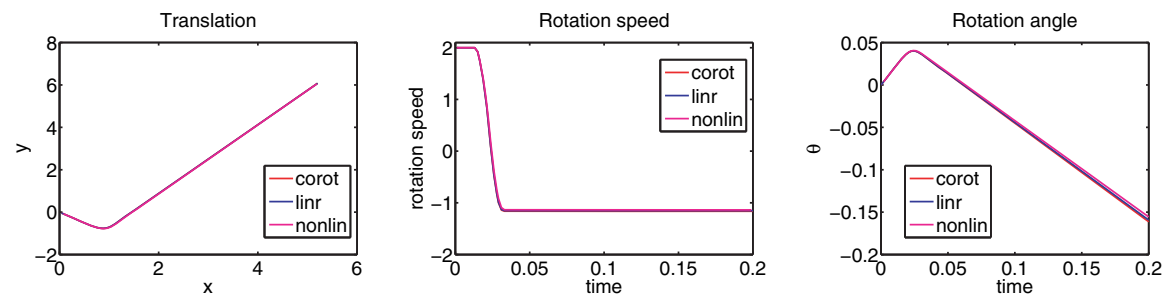

FIG. 6.8. Ball on plane with friction. Translation (left), angular velocity (middle), rotation angle for corotational, linearized $r$ and standard nonlinear scheme (right).

tational scheme without linearization and the nonlinear scheme give similar results, while the scheme with linearized $r$ exhibits discrepancy. The three schemes are energy consistent. Additionally, we compare the translation, angular velocity, and rotation angle; see Figure 6.8. Due to friction, the rotation direction reverses during contact. The results for the three schemes almost coincide. We see a slightly larger average rotation velocity after contact when using the standard nonlinear scheme than for the corotational schemes. This results in small differences of the rotation angle.

6.4. Ball and ellipse between two planes - multiple contact problem. In our second example, we use a similar setting. Here a ball moves between two planes and comes into contact with both planes. The domain $\Omega$ is a ball of radius $r=1$; see Figure 6.9, the initial distance is 0.5 between the center of the ball and the upper plane, and 0.1 between the center and the lower plane. As material parameters, we use $E=1.62 \cdot 10^{7}, \nu=0.2$, and $\rho=2$. The initial conditions are given by $\dot{\theta}_{0}=20$ and $\dot{T}_{0}=V_{0}=(40,-40)^{\top}$; as a time step we choose $\Delta t=0.001$. As before, we use Coulomb friction with a friction coefficient $\mathfrak{F}=0.2$.

Here, we compare our two corotational schemes (with and without the linearization of $r$ ). The solution at various time steps is plotted in Figure 6.10. The results for the mean translation, angular velocity, and the angle of rotation can be seen in Figure 6.11. First of all, we see that the dynamic behavior is much more complex than in 

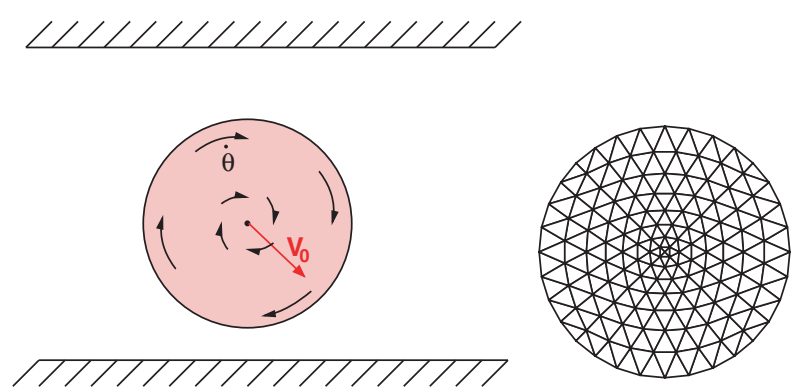

FIG. 6.9. Ball between two planes. Left: Problem setting. Right: Grid.
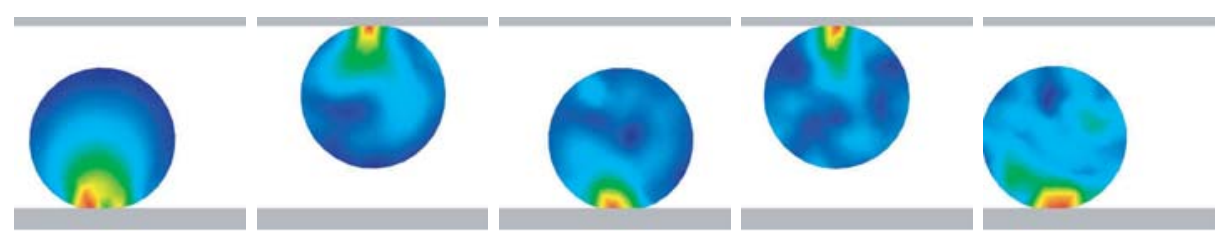

FIG. 6.10. Ball between two planes. Deformed body and effective stress at time $t=0.003,0.022$, $0.041,0.07$, and 0.095 .
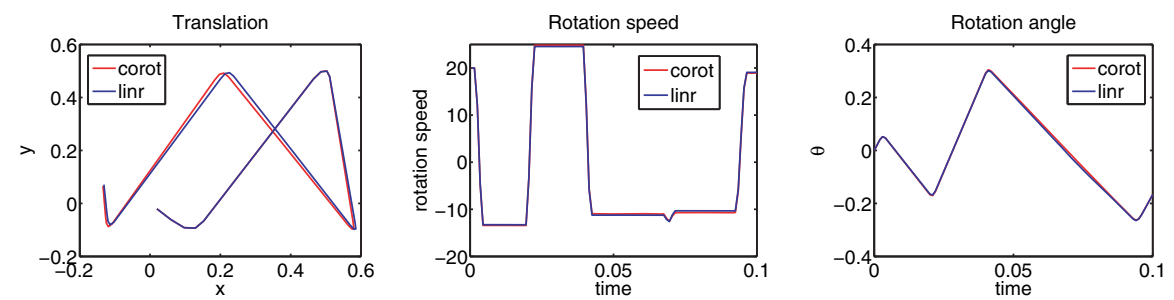

FIG. 6.11. Ball between two planes. Translation (left), angular velocity (middle), rotation angle for standard corotational and linearized $r$ scheme (right).

the single-contact problem. Nevertheless, our corotational schemes are able to handle the large rotation and all contact situations. All five contacts can be seen in the first picture of Figure 6.11. The contact angles vary between contact times. This has also an effect on the change of the rotation velocity as can be seen in the second graph. The energy and angular momentum is plotted in Figure 6.12. Again, both schemes are energy consistent and yield similar results. This can also be observed in the evolution of the mean translation and the mean rotation. However, small differences in the translation occur.

In the next example, we demonstrate that even small differences can have a strong influence on the long-time behavior of dynamical contact problems. Instead of a ball, we use now an ellipse with the same material parameters and initial data as for the ball. The problem setting and initial grid is depicted in Figure 6.13. We note that this problem is ill posed in the following sense. A small change in the initial conditions leads to a different contact situation. Impact after impact, this small difference amplifies, and a completely different behavior occurs. The solution at various time steps is plotted in Figure 6.14.

The results comparing our corotational schemes are given in Figure 6.15. For a long time in the interval $(0,0.8)$, results groove to be identical. Only small differences 

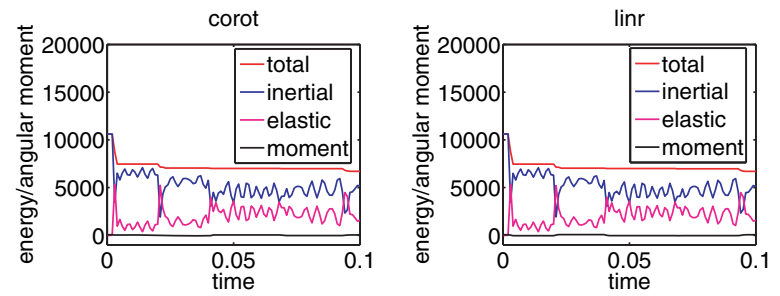

FIG. 6.12. Ball between two planes. Energies and angular momentum for corotational (left), linearized $r$ (middle), and standard nonlinear scheme (right).
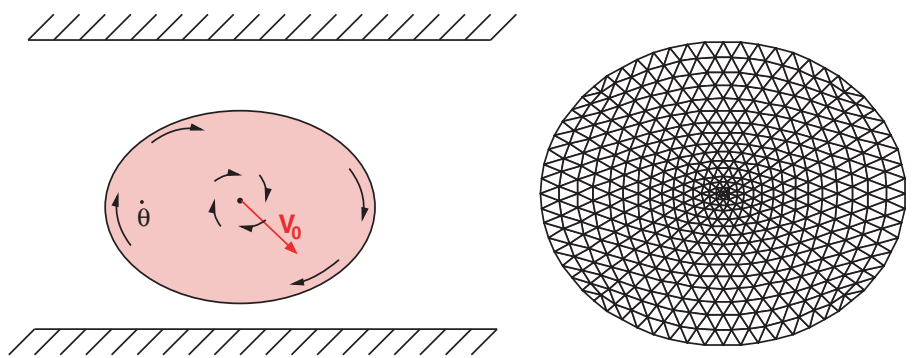

FIG. 6.13. Ellipse between two planes. Left: Problem setting. Right: Grid.
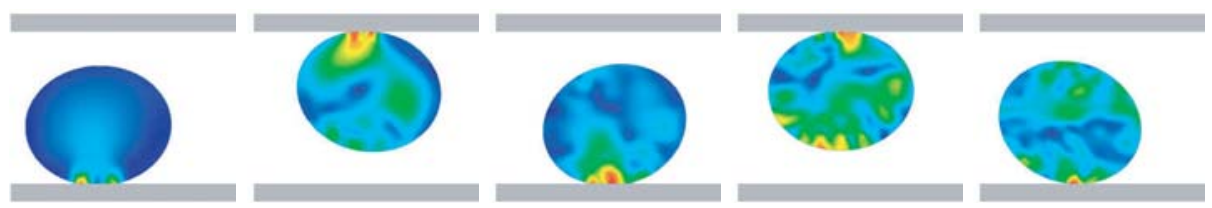

FIG. 6.14. Ellipse between two planes. Deformed body and effective stress at time $t=0.004$, $0.025,0.045,0.071$, and 0.099 .
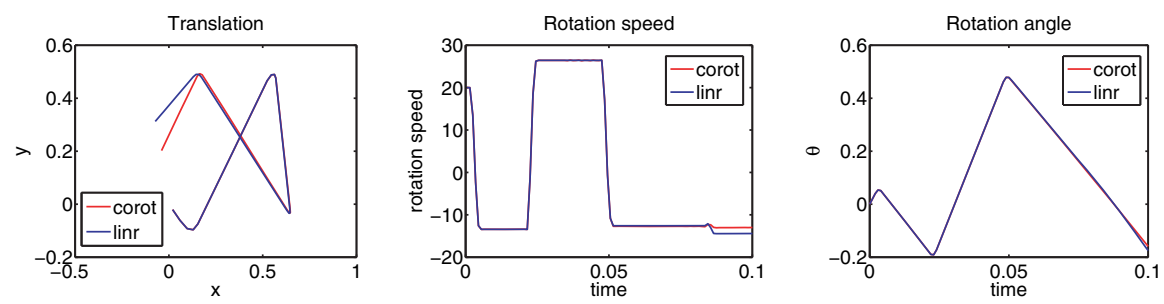

FIG. 6.15. Ellipse between two planes. Translation (left), angular velocity (middle), rotation angle for standard corotational and linearized $r$ scheme (right).

in the rotation velocity can be observed after the third contact. However, this small difference leads to a completely different mean translation and thus rotation velocity after the fourth contact.

6.5. Torus on plane $-3 D$ contact example. In the last example, we apply our corotational scheme to a $3 D$ problem. Here, the domain is given by a torus with inner radius 6 and outer radius 8; see Figure 6.16. The material parameters are given by $E=1.6 \cdot 10^{5}, \nu=0.2$, and $\rho=1$. The torus is assumed to impact a plane located at distance 9 from the initial location of its center. As an initial condition, we use 

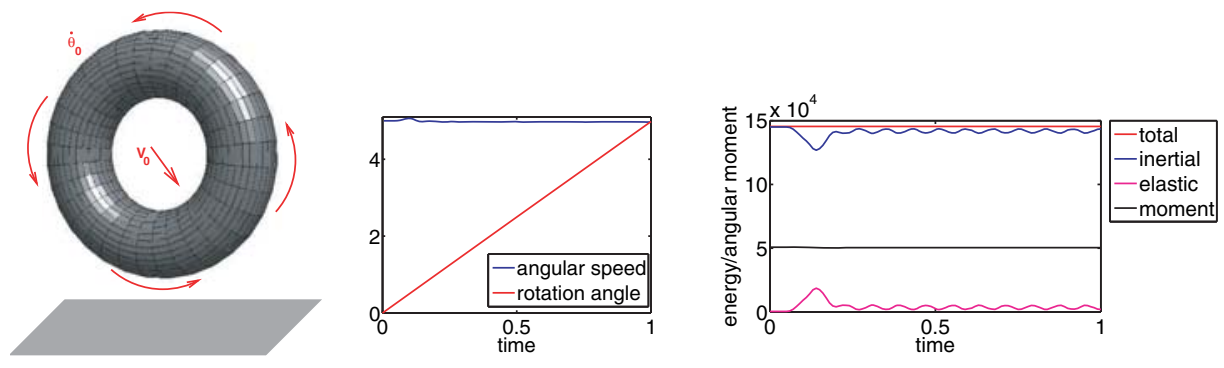

FIG. 6.16. Torus on plane. Problem setting (left), angular speed and rotation angle (middle), eEnergies and angular momentum (right).
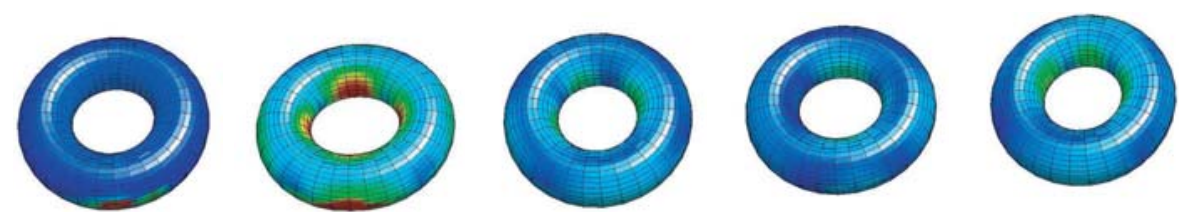

FiG. 6.17. Torus on plane. Deformed mesh at time $t=0.05,0.15,0.25,0.35$, and 0.45 .
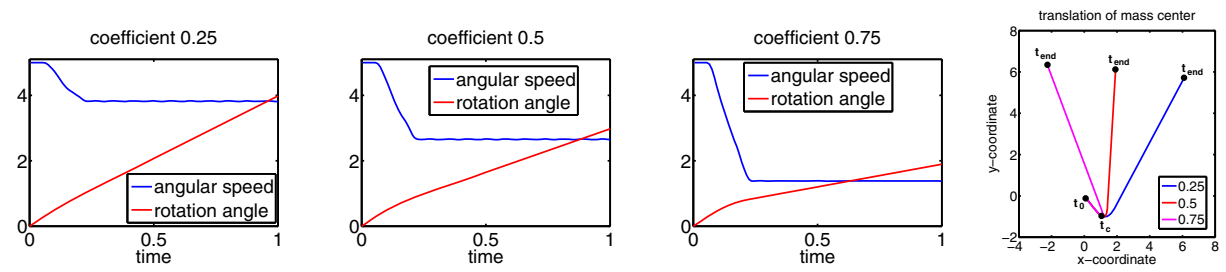

FIG. 6.18. Torus on plane with friction. The change of angular velocity and rotation angle for different friction coefficients $\mathcal{F}$ (left), influence of $\mathcal{F}$ on the translation after time of first contact ( $\left.t_{c}\right)$ (right).

$\dot{\theta}_{0}=5$ and $\dot{T}_{0}=V_{0}=(10,-10)^{\top}$; as a time step, we choose $\Delta t=0.01$. In Figure 6.17 , the deformed mesh at various time steps is depicted for the frictionless case. The angular velocity and energy plots are given in the last two pictures of Figure 6.16. The results are similar to those from the first example. Additionally, we consider the case of Coulomb friction and test the influence of the friction parameter $\mathcal{F}$ on the change of rotation speed during contact. In Figure 6.18, the results for $\mathcal{F}=0.25,0.5$, and 0.75 are given. The higher the friction is, the smaller the angular speed is after contact. As can be expected, the friction coefficient also influences the flight direction after contact; see the right picture in Figure 6.18.

7. Conclusion. We have introduced two energy-conserving corotational formulations for bodies undergoing large displacements and small deformation in the presence of frictional contact. Unlike standard schemes, these formulations strictly preserve rigid motions independently of the time step, and the first one also preserves angular momentum at the price of solving a nonlinear problem on the rotation angle. Numerical tests demonstrate the efficiency and the accuracy of the proposed approach as compared to the standard conservative approach. 
Acknowledgment. P. Hauret is strongly indebted to Michael Ortiz for a great postdoctoral experience at Caltech.

\section{REFERENCES}

[1] F. ARmero AND E. PetöCZ, Formulation and analysis of conserving algorithms for frictionless dynamic contact/impact problems, Comput. Methods Appl. Mech. Engrg., 158 (1998), pp. 269-300.

[2] M. Віот, The Mechanics of Incremental Deformations, McGraw-Hill, New-York, 1962.

[3] V. Chawla and T. Laursen, Energy consistent algorithms for frictional contact problems, Internat. J. Numer. Methods Engrg., 42 (1998), pp. 799-827.

[4] P.-G. Ciarlet, Mathematical Elasticity, North-Holland, Amsterdam, 1988.

[5] M. CRisfield, A consistent corotational formulation for nonlinear three-dimensional beam elements, Comput. Methods Appl. Mech. Engrg., 81 (1990), pp. 131-150.

[6] M. Crisfield, Nonlinear Finite Element Analysis of Solids and Structures, Vol. 2, Advanced Topics, John Wiley, New York, 1997.

[7] B. F. DE Veubeke, The dynamics of flexible bodies, Internat. J. Engrg. Sci., 14 (1976), pp. 895913.

[8] C. Eck, J. Jarušek, And M. Krbec, Unilateral Contact Problems: Variational Methods and Existence Theorems, CRC Press, Boca Raton, FL, 2005.

[9] C. Felippa And B. Haugen, A unified formulation of small-strain corotational finite elements: I. Theory, Comput. Methods Appl. Mech. Engrg., 194 (2005), pp. 2285-2335.

[10] C. Grandmont, Y. Maday, And P. Metier, Modeling and analysis of an elastic problem in large displacements and small strains, J. Elasticity, 87 (2007), pp. 29-72.

[11] P. Hauret and P. L. Tallac, Energy-controlling time integration methods for nonlinear elastodynamcs and low-velocity impact, Comput. Methods Appl. Mech. Engrg., 195 (2006), pp. $4890-4916$.

[12] P. HAURet, Méthodes numériques pour la dynamique des structures non-linéaires incompressibles à deux échelles (Numerical Methods for the Dynamic Analysis of Two-Scale Incompressible Nonlinear Structures), Ph.D. thesis, Ecole Polytechnique, Paris, 2004.

[13] M. Hintermüller, K. Ito, AND K. Kunisch, The primal-dual active set strategy as a semismooth Newton method, SIAM J. Optim., 13 (2002), pp. 865-888.

[14] S. Hüeber, G. Stadler, And B. Wohlmuth, A primal-dual active set algorithm for threedimensional contact problems with Coulomb friction, SIAM J. Sci. Comput., 30 (2008), pp. $572-596$.

[15] S. Hüeber And B. Wohlmuth, A primal-dual active set strategy for non-linear multibody contact problems, Comput. Methods Appl. Mech. Engrg., 194 (2005), pp. 3147-3166.

[16] N. Kikuchi and J. Oden, Contact Problems in Elasticity: A Study of Variational Inequalities and Finite Element Methods, SIAM Studies in Applied Mathematics Vol. 8, SIAM, Philadelphia, 1988.

[17] T. Launsen and V. Chawla, Design of energy conserving algorithms for frictionless dynamic contact problems, Internat. J. Numer. Methods Engrg., 40 (1997), pp. 836-886.

[18] T. Laursen AND G. R. Love, Improved implicit integrators for transient impact problemsGeometric admissibility within the conserving framework, Internat. J. Numer. Methods Engrg., 52 (2002), pp. 245-274.

[19] T. Laursen, Computational Contact and Impact Mechanics, Springer, Berlin, 2002.

[20] B. Nour-OMid AND C. RANKIn, Finite rotation analysis and consistent linearizations using projectors, Comput. Methods Appl. Mech. Engrg., 93 (1991), pp. 353-384.

[21] M. Nygard and P. Bergan, Advances in treating large rotations for nonlinear problems, in State of the Art Surveys on Computational Mechanics, A. Noor and J. Oden, eds., American Society of Mechanical Engineers, New York, 1989, pp. 305-332.

[22] C. Rankin and F. BRognan, An element-independent corotational procedure for the treatment of large rotations, ASME J. Pressure Vessel Tech., 108 (1986), pp. 165-174.

[23] C. Rankin And B. Nour-Omid, The use of projectors to improve finite element performance, Comput. \& Structures, 30 (1988), pp. 257-267.

[24] J. Salomon, A. Weiss, and B. Wohlmuth, Energy conserving algorithms for a corotational formulation, SIAM J. Numer. Anal., 46 (2008), pp. 1842-1866.

[25] K. Willner, Kontinuums- und Kontaktmechanik, Springer, Berlin, 2003.

[26] B. Wohlmuth, A mortar finite element method using dual spaces for the Lagrange multiplier, SIAM J. Numer. Anal., 38 (2000), pp. 989-1012.

[27] P. Wriggers, Computational Contact Mechanics, John Wiley, New York, 2002.

Copyright (c) by SIAM. Unauthorized reproduction of this article is prohibited. 